\title{
Cantor Type Basic Sets of Surface A-endomorphisms
}

\author{
V.Z. Grines, E. V.Zhuzhoma
}

The paper is devoted to an investigation of the genus of an orientable closed surface $M^{2}$ which admits $A$-endomorphisms whose nonwandering set contains a one-dimensional strictly invariant contracting repeller $\Lambda_{r}$ with a uniquely defined unstable bundle and with an admissible boundary of finite type. First, we prove that, if $M^{2}$ is a torus or a sphere, then $M^{2}$ admits such an endomorphism. We also show that, if $\Omega$ is a basic set with a uniquely defined unstable bundle of the endomorphism $f: M^{2} \rightarrow M^{2}$ of a closed orientable surface $M^{2}$ and $f$ is not a diffeomorphism, then $\Omega$ cannot be a Cantor type expanding attractor. At last, we prove that, if $f: M^{2} \rightarrow M^{2}$ is an $A$-endomorphism whose nonwandering set consists of a finite number of isolated periodic sink orbits and a one-dimensional strictly invariant contracting repeller of Cantor type $\Omega_{r}$ with a uniquely defined unstable bundle and such that the lamination consisting of stable manifolds of $\Omega_{r}$ is regular, then $M^{2}$ is a two-dimensional torus $\mathbb{T}^{2}$ or a two-dimensional sphere $\mathbb{S}^{2}$

Keywords: A-endomorphism, regular lamination, attractor, repeller, strictly invariant set

\section{Introduction and main results}

By an endomorphism we mean a $C^{1}$-smooth surjective map of a manifold to itself. If an endomorphism $f$ is a one-to-one map and the inverse mapping $f^{-1}$ is $C^{1}$-smooth, then $f$ becomes a diffeomorphism. We see that the notion of endomorphism generalizes the notion of diffeomorphism.

We denote by $\operatorname{End}(M)$ the space of endomorphisms of a closed manifold $M$ endowed with the standard $C^{1}$-topology. Let $g \in \operatorname{End}(M)$. The orbit or $g$-orbit of the point $x_{0} \in M$ is a countable or finite set $\left\{x_{i}\right\}_{i=-\infty}^{\infty}=O\left(x_{0}\right)$ such that $g\left(x_{i}\right)=x_{i+1}$ for any $i \in \mathbb{Z}$. The set $\left\{x_{i}\right\}_{i=0}^{\infty}=O^{+}\left(x_{0}\right) \subset O\left(x_{0}\right)$ is called the positive semi-orbit of the point $x_{0}$. A positive semi-orbit

Received July 30, 2021

Accepted August 25, 2021

This work is supported by the Russian Science Foundation under grant 17-11-01041.

Vyacheslav Z. Grines

vgrines@yandex.ru

Evgeny V. Zhuzhoma

zhuzhoma@mail.ru

National Research University Higher School of Economics

ul. B. Pecherskaya 25/12, Nizhny Novgorod, 603150 Russia

RUSSIAN JOURNAL OF NONLINEAR DYNAMICS, 2021, 17(3), 335-345 
is uniquely defined by the original point $x_{0}$, while the set of orbits passing through $x_{0}$ (which we denote $\left.Q\left(x_{0}\right)\right)$ is generally a set of cardinality continuum. To a fixed element $\alpha \in Q\left(x_{0}\right)$ there is uniquely appropriated the orbit $\left\{x_{i}^{\alpha}\right\}_{-\infty}^{\infty}=O^{\alpha}\left(x_{0}\right)$, where $g\left(x_{i}^{\alpha}\right)=x_{i+1}^{\alpha}$ for any $i \in \mathbb{Z}$ and $x_{0}^{\alpha}=$ $=x_{0}$. The set $\left\{x_{i}^{\alpha}\right\}_{-\infty}^{0}=O^{\alpha-}\left(x_{0}\right)$ is called the negative semi-orbit of the orbit $O^{\alpha}\left(x_{0}\right)$. Below for simplicity we will omit index $\alpha$ when we consider an orbit of the point $x_{0}$. A point $x \in M$ of the endomorphism $f: M \rightarrow M$ is called nonwandering if for any neighborhood $U$ of a point $x$ there is $i>0$ such that $f^{i}(U) \cap U \neq \varnothing$. The set of nonwandering points forms the nonwandering set of the endomorphism $f$ and is denoted by $N W(f)$. It is known that a nonwandering set is always forward-invariant, that is, $f(N W(f)) \subset N W(f)$.

An orbit $O\left(x_{0}\right)$ is called hyperbolic if there is a continuous splitting of the tangent space

$$
\mathbb{T}_{O\left(x_{0}\right)} M=\bigcup_{i=-\infty}^{\infty} \mathbb{T}_{x_{i}} M=\mathbb{E}^{s} \oplus \mathbb{E}^{u}=\bigcup_{i=-\infty}^{\infty} \mathbb{E}_{x_{i}}^{s} \oplus \mathbb{E}_{x_{i}}^{u}
$$

which is invariant with respect to $D g$ and such that

$$
\begin{gathered}
0<\left\|D g^{m}(v)\right\| \leqslant c \mu^{m}\|v\|, \quad\left\|D g^{m}(w)\right\| \geqslant c^{-1} \mu^{-m}\|w\| \\
\text { where } v \in \mathbb{E}^{s} \backslash\{0\}, \quad w \in \mathbb{E}^{u}, \quad \forall m \in \mathbb{N}
\end{gathered}
$$

for some constants $c>0,0<\mu<1$ and a Riemannian metric on $\mathbb{T} M$.

Note that the unstable subbundle $\mathbb{E}^{u}\left(x_{0}\right)$ depends, in general, on the negative semi-orbit $\left\{x_{i}\right\}_{i=-\infty}^{0}$, so we will write $\mathbb{E}^{u}\left(x_{0},\left\{x_{i}\right\}_{i=-\infty}^{0}\right)$. It may be $\mathbb{E}^{u}\left(x_{0},\left\{x_{i}\right\}_{i=-\infty}^{0}\right) \neq \mathbb{E}^{u}\left(y_{0},\left\{y_{i}\right\}_{i=-\infty}^{0}\right)$ for $x_{0}=y_{0}$ with $\left\{x_{i}\right\}_{-\infty}^{0}=O^{-}\left(x_{0}\right) \neq O^{-}\left(y_{0}\right)=\left\{y_{i}\right\}_{-\infty}^{0}$. Such an effect is impossible for a stable subbundle $\mathbb{E}^{S}\left(x_{0}\right)$ that depends only on the point $x_{0}$.

The set $\Lambda \subset M$ is called hyperbolic if $f(\Lambda)=\Lambda$, any orbit lying in $\Lambda$ is hyperbolic and the constants $c>0,0<\mu<1$ in the above estimates do not depend on the choice of the orbit (therefore, one sometimes talks about uniform hyperbolicity). Note that the equality $f(\Lambda)=\Lambda$ guarantees that for any point $x_{0} \in \Lambda$ there is at least one negative semi-orbit lying in $\Lambda$.

By analogy with a diffeomorphism satisfying the Smale axiom $A$, an endomorphism $f: M \rightarrow$ $\rightarrow M$ is called an A-endomorphism if its nonwandering set $N W(f)$ is hyperbolic and periodic points are dense in $N W(f)$. Recall that a map $N \rightarrow N$ is called transitive if there exists a point $x \in$ whose positive semi-orbit is dense in $N$.

In [19], the Spectral Theorem for an A-endomorphisms is proved, which is a generalization of the Spectral Theorem by Smale [21] for A-diffeomorphisms. According to [19], the nonwandering set $N W(f)$ of the A-endomorphism $f: M \rightarrow M$ is represented in a unique way up to numbering as a union of closed and pairwise disjoint sets

$$
N W(f)=\Omega_{1} \cup \ldots \cup \Omega_{l}, \quad \Omega_{i} \cap \Omega_{j}=\varnothing \quad \text { for } i \neq j,
$$

such that $f\left(\Omega_{j}\right)=\Omega_{j}$ and $\left.f\right|_{\Omega_{j}}: \Omega_{j} \rightarrow \Omega_{j}$ is transitive for all $1 \leqslant j \leqslant l$. The sets $\Omega_{1}, \ldots, \Omega_{l}$ are called basic sets.

To date, there are a number of complete classification results for one-dimensional basic sets of A-diffeomorphisms on closed surfaces $M^{2}[2-5,11]$ and manifolds of higher dimension $[6,7$, $12,13]$. It is proved that one-dimensional basic sets on $M^{2}$ are Cantor laminations, that is, each basic set $\Omega$ is the union of pairwise disjoint curves locally arranged as a direct product of a segment and the Cantor set. Moreover, each leaf of such a lamination is homeomorphic in the intrinsic topology to the line $\mathbb{R}$, and the admissible boundary from the interior of any connected component of the set $M^{2} \backslash \Omega$ consists of a finite number of periodic leaves.

For A-endomorphisms, which are not A-diffeomorphisms, there are few classes of systems for which it is possible to describe the structure of basic sets and obtain complete classification 
results. Such classes include interval and circle endomorphisms, endomorphisms arising in complex dynamics on the Riemann sphere, and expanding endomorphisms of manifolds of higher dimension [20]. It should be noted that the structure of the basic sets of A-endomorphisms has not yet been studied so exhaustively even for endomorphisms of surfaces.

In this paper, we consider one-dimensional strictly hyperbolic basic sets of $A$-endomorphisms of closed surfaces. From the point of view of applications, an important role is played by basic sets which are attractors and repellers. We investigate the topology of orientable close surfaces which admits $A$-endomorphisms with such basic sets.

Let us start presenting the main results. A hyperbolic set $\Lambda$ is called uniquely hyperbolic, or a set with a uniquely defined unstable bundle, if the unstable subbundle $\mathbb{E}^{u}\left(x_{0},\left\{x_{i}\right\}_{i=-\infty}^{0}\right)=\mathbb{E}^{u}\left(x_{0}\right)$ does not depend on the negative semi-orbit $\left\{x_{i}\right\}_{i=-\infty}^{0}$ for any point $x_{0} \in \Lambda$.

A basic set $\Omega_{a}$ is called an attractor if there exists a neighborhood $U$ of the set $\Omega_{a}$ such that

$$
f(\operatorname{clos} U) \subset U, \bigcap_{i \geqslant 0} f^{i}(U)=\Omega_{a} .
$$

An attractor $\Omega_{a}$ is called expanding if its topological dimension is equal to the dimension of the unstable subbundle $\mathbb{E}^{u}\left(x_{0},\left\{x_{i}\right\}_{i=-\infty}^{0}\right)$ for any point $x_{0} \in \Omega_{a}$ and any negative semiorbit $\left\{x_{i}\right\}_{i=-\infty}^{0} \subset \Omega_{a}$.

A basic set $\Omega_{r}$ is called a repeller if there exists a neighborhood $U$ of the set $\Omega_{r}$ such that

$$
\operatorname{clos} U \subset f(U), \bigcap_{i \geqslant 0} f^{-i}(U)=\Omega_{r} .
$$

A repeller $\Omega_{r}$ is called contracting if its topological dimension is equal to the dimension of the stable subbundle $\mathbb{E}^{s}\left(x_{0}\right)$ for any point $x_{0} \in \Omega_{r}$.

A basic set $\Omega$ is called strictly invariant if $f^{-1}(\Omega)=\Omega=f(\Omega)$. It follows directly from the definition of hyperbolicity that, in a certain neighborhood of the basic set, an endomorphism is a local diffeomorphism. Below, saying that an endomorphism is not a diffeomorphism, we will assume for simplicity that an endomorphism is a finitely multiple cover of a multiplicity at least two, with the exception of a finite number of branching points.

Let $K$ be an open set of a topological space $N$, and $\partial K$ be the boundary of the set $K$. A subset of $\delta(K) \subset \partial K$ is called the admissible boundary from $K$ (sometimes one says, admissible boundary from interior $K$ ) if for each point $z \in \partial K$ there exists an arc $d$ with the end point $z$ such that $d \backslash\{z\} \subset K$.

Let $\Omega$ be a strictly invariant one-dimensional basic set with a uniquely defined unstable bundle of an $A$-endomorphism $f: M^{2} \rightarrow M^{2}$. It follows from the closeness of the set $\Omega$ and Proposition 2.7 [15] that, if $\Omega$ is an expanding attractor, then unstable manifolds of points from $\Omega$ form the lamination $\mathcal{L}(\Omega)=\bigcup_{x \in \Omega} W^{u}(x)$. Similarly, if $\Omega$ is a contracting repeller, then its stable manifolds also form a lamination, which we will denote by the same symbol $\mathcal{L}$.

We will say that $\mathcal{L}(\Omega)$ is a Cantor lamination with an admissible boundary of finite type if the following conditions hold:

1) all leaves of lamination $\mathcal{L}(\Omega)$ are the images of injective immersion of the line $\mathbb{R}$, and the union of leaves of $\mathcal{L}(\Omega)$ is locally homeomorphic to the product of a segment and Cantor set;

2) for any component $K$ of the set $M^{2} \backslash \mathcal{L}(\Omega)$ the boundary $\delta(K)$ admissible from $K$ consists of a finite number of invariant manifolds (unstable manifolds if $\Omega$ is an expanding attractor, and stable manifolds if $\Omega$ is a contracting repeller). 
We will say that $\Omega$ is a basic set of Cantor type with an admissible boundary of finite type if $\mathcal{L}(\Omega)$ is a Cantor lamination with an admissible boundary of finite type.

Denote by $\mathbb{T}^{2}$ the two-dimensional torus, and by $\mathbb{S}^{2}$, the two-dimensional sphere.

Theorem 1. There is an endomorphism $f: M^{2} \rightarrow M^{2}$, where $M^{2}=\mathbb{T}^{2}$ or $M^{2}=\mathbb{S}^{2}$, such that the nonwandering set of $f$ contains a one-dimensional strictly invariant contracting repeller $\Omega_{r}$ with uniquely defined unstable bundle and with an admissible boundary of finite type.

It is known that on any closed surface $M^{2}$ there is an A-diffeomorphism $f: M^{2} \rightarrow M^{2}$ with a one-dimensional expanding attractor and a one-dimensional contracting repeller of Cantor type (in both cases, the basic sets have an admissible boundary of finite type). The following statement contrasts with the situation where an endomorphism is not a diffeomorphism, even if the attractor of the endomorphism is strictly invariant and has a uniquely defined unstable bundle (for a diffeomorphism, this is done automatically).

Theorem 2. Let $\Omega$ be a basic set with a uniquely defined unstable bundle of endomorphism $f: M^{2} \rightarrow M^{2}$ of a closed orientable surface $M^{2}$, and suppose $f$ is not a diffeomorphism. Then $\Omega$ cannot be a Cantor expanding attractor with an admissible boundary of finite type.

The A-endomorphisms satisfying Theorem 1 on the two-dimensional torus $\mathbb{T}^{2}$ and the twodimensional sphere $\mathbb{S}^{2}$ have nonwandering sets consisting of a finite number of isolated sinks and a one-dimensional strictly invariant contracting repeller of Cantor type. In addition, the laminations corresponding to one-dimensional repellers continue on $\mathbb{S}^{2}$ to foliations with a finite number of singularities of a nonzero index, and on $\mathbb{T}^{2}$ to foliations without singularities. It is natural to consider the existence of A-endomorphisms with similar properties on other closed surfaces. Note that the index of a singularity of foliation in the component $C$ of the set $M^{2} \backslash \mathcal{L}\left(\Lambda_{r}\right)$ was related to the number of stable manifolds belonging to the admissible boundary of $C$ as follows.

Let $\nu$ be the number of leaves (stable manifolds) in the boundary of the component $C$ admissible from $C$. Then in $C$ the foliation has the singularity of the index $1-\frac{\nu}{2}$. On $\mathbb{S}^{2}$, four components of the set $\mathbb{S}^{2} \backslash \Omega$ have exactly one admissible leaf. Therefore, in each such component the singularity has the index $\frac{1}{2}$. On $\mathbb{T}^{2}$, according to the construction, $\nu=2$ for all components of the set $\mathbb{T}^{2} \backslash \Omega$. Therefore, there are no singularities in each component.

We call the lamination $\mathcal{L}$ regular if it continues to a foliation $\mathcal{F}$ with the following property. Let $C$ be a component of the set $M^{2} \backslash \mathcal{L}$, and $\delta(C)$ be its boundary admissible from $C$, consisting of $\nu$ leaves. Then $\mathcal{F}$ has exactly one singularity of the index $1-\frac{\nu}{2}$ in $C$ provided $\nu \neq 2$. If $\nu=2$, then there are no singularities in $C$. The following statement holds.

Theorem 3. Let $f: M^{2} \rightarrow M^{2}$ be an A-endomorphism on a closed orientable surface $M^{2}$, such that the nonwandering set $N W(f)$ consists of a finite number of isolated periodic sink orbits and a one-dimensional strictly invariant contracting repeller of Cantor type $\Lambda_{r}$ with a uniquely defined unstable bundle. Suppose that the lamination $\mathcal{L}\left(\Lambda_{r}\right)$ is regular. Then $M^{2}$ is the twodimensional torus $\mathbb{T}^{2}$ or the two-dimensional sphere $\mathbb{S}^{2}$.

Note that the analog of Theorem 2 does not hold, in general, for endomorphisms of threedimensional manifolds. Indeed, consider a 3-dimensional torus $\mathbb{T}^{3}$, which we represent as the product of $\mathbb{T}^{2} \times \mathbb{S}^{1}$ of the two-dimensional torus $\mathbb{T}^{2}$ and the circle $\mathbb{S}^{1}$. On the first multiplier $\mathbb{T}^{2}$, we define a $D A$-diffeomorphism $f_{0}: \mathbb{T}^{2} \rightarrow \mathbb{T}^{2}$ with an isolated source and a nontrivial onedimensional expanding attractor $\Lambda_{1}$, and on the second multiplier $\mathbb{S}^{1}$, we define the expanding endomorphism $E_{2}: \mathbb{S}^{1} \rightarrow \mathbb{S}^{1}$ of the form $x \mapsto 2 x \bmod 1$. The endomorphism $(z, t) \rightarrow\left(f_{0}(z) ; E_{2}(t)\right)$, $z \in \mathbb{T}^{2}, t \in \mathbb{S}^{1}$, has the expanding strictly invariant two-dimensional attractor $\Lambda_{1} \times \mathbb{S}^{1}$ with a uniquely defined unstable Cantor type bundle, that is, $\Lambda_{1} \times \mathbb{S}^{1}$ is locally homeomorphic to the 
product of a Cantor set on a two-dimensional plane. At the same time, each connected component of the set $\mathbb{T}^{3} \backslash\left(\Lambda_{1} \times \mathbb{S}^{1}\right)$ has a boundary admissible from $\mathbb{T}^{3} \backslash\left(\Lambda_{1} \times \mathbb{S}^{1}\right)$ consisting of two lamination leaves $\mathcal{L}\left(\Lambda_{1} \times \mathbb{S}^{1}\right)$, that is, an expanding attractor $\Lambda_{1} \times \mathbb{S}^{1}$ has an admissible boundary of finite type.

Let us give some other examples of one-dimensional attractors and repellers of endomorphisms of two-dimensional manifolds. Let $h: \mathbb{S}^{1} \rightarrow \mathbb{S}^{1}$ be a Morse-Smale diffeomorphism with one source $s_{r}$ and one sink $s_{a}$. Then the nonwandering set of the endomorphism $\left(h ; E_{2}\right): \mathbb{S}^{1} \times \mathbb{S}^{1}=$ $=\mathbb{T}^{2} \rightarrow \mathbb{T}^{2}$ contains the strictly invariant one-dimensional expanding attractor $\left\{s_{a}\right\} \times \mathbb{S}^{1}$ with a uniquely defined unstable bundle and a strictly invariant one-dimensional repeller $\left\{s_{r}\right\} \times \mathbb{S}^{1}$ with a uniquely defined unstable bundle (both are homeomorphic to $\mathbb{S}^{1}$ ). Note that the repeller is not strictly contacting, since its stable bundle is zero-dimensional.

The nonwandering set of the endomorphism $S H: \mathbb{S}^{1} \rightarrow \mathbb{S}^{1}$ constructed in [20] consists of an isolated sink $s_{1}$ and a nontrivial (zero-dimensional) repeller $\Sigma_{r}$, homeomorphic to the Cantor set $C$. Then the nonwandering set of the endomorphism $\left(S H ; E_{2}\right): \mathbb{S}^{1} \times \mathbb{S}^{1}=\mathbb{T}^{2} \rightarrow \mathbb{T}^{2}$ consists of a one-dimensional expanding attractor $\left\{s_{1}\right\} \times \mathbb{S}^{1}$, which is not strictly invariant, and a onedimensional repeller $R$ which is noncontracting and is homeomorphic to $C \times \mathbb{S}^{1}$. Both basic sets have uniquely defined unstable bundles.

There are repellers that are not submanifolds. It is known, for example, that endomorphisms of surfaces arising in complex dynamics can have one-dimensional repellers having a fractal structure.

The structure of the article is as follows. Section 2 provides preliminary information and proves auxiliary results. The main theorems are proved in Section 3.

\section{Auxiliary results}

Let $\Lambda$ be the hyperbolic set of a $C^{k}$-endomorphism $f: M \rightarrow M(k \geqslant 1)$, and let $p \in \Lambda$. It is known $[15,18,19]$ that for a sufficiently small $\delta>0$ set

$$
W_{\delta, l o c}^{s}(p)=\left\{x \in M: \varrho\left(f^{i}(p) ; f^{i}(x)\right)<\delta \forall i \geqslant 0\right\}
$$

is a $C^{k}$-embedded open disk $\mathbb{B}^{\operatorname{dim} \mathbb{E}^{s}(p)}$ with the tangent space $\mathbb{E}^{s}(p)$ at the point $p$, and for any point $x \in W_{\delta, l o c}^{s}(p)$

$$
\varrho\left(f^{i}(p) ; f^{i}(x)\right) \longrightarrow 0 \text { exponentially fast as } i \rightarrow \infty .
$$

The set $W_{\delta, l o c}^{s}(p)$ is called a local stable manifold at the point $p$. A globally stable manifold (or, simply, a stable manifold) at the point $p$ is called a set

$$
W^{s}(p)=\left\{x \in M: \varrho\left(f^{i}(p) ; f^{i}(x)\right) \rightarrow 0 \text { as } i \rightarrow \infty\right\} .
$$

If $\Lambda$ is a compact hyperbolic set, then the number $\delta$ can be taken so that local stable manifolds $W_{\delta, l o c}^{s}(z)$ are defined for all points $z \in \Lambda$. Then the global stable manifold $W^{s}(p)$ can be represented as follows:

$$
W^{s}(p)=\bigcup_{i \geqslant 0} \operatorname{Comp}_{p} f^{-i}\left[W_{\delta, l o c}^{s}\left(p_{i}\right)\right], \text { where } p_{i}=f^{i}(p)
$$

where $\operatorname{Comp}_{z} K$ denotes the connected component of the set $K$ containing $z$.

RUSSIAN JOURNAL OF NONLINEAR DYNAMICS, 2021, 17(3), 335-345 — \#) 
Unlike stable bundles $\mathbb{E}^{s}$, unstable bundles depend not only on the point, but also on the negative half-orbit of the point. Let $O^{-}(p) \subset \Lambda$ be the negative semi-orbit of the point $p \in \Lambda$. According to $[15,18,19]$, for a sufficiently small $\delta>0$ set

$$
W_{\delta, l o c}^{u}\left(O^{-}(p)\right)=\left\{x \in M: \exists O^{-}(x), \varrho\left(p_{-i} ; x_{-i}\right)<\delta \forall i \geqslant 0 \text {, where } p_{-i} \in O^{-}(p), x_{-i} \in O^{-}(x)\right\}
$$

is a $C^{k}$-embedded open disk $\mathbb{B}^{\operatorname{dim} \mathbb{E}^{u}\left(O^{-}(p)\right)}$ with a tangent space, which we will denote by $\mathbb{E}^{u}\left(O^{-}(p)\right)$, at the point $p$. Moreover, for any point above $x \in W_{\delta, l o c}^{u}\left(O^{-}(p)\right)$

$$
\varrho\left(p_{-i} ; x_{-i}\right) \rightarrow 0 \text { exponentially fast as } i \rightarrow \infty .
$$

The set $W_{\delta, l o c}^{u}\left(O^{-}(p)\right)$ is called a local unstable manifold with respect to the negative semiorbit $O^{-}(p)$ at the point $p$. A globally unstable manifold (or, simply, an unstable manifold) with respect to the negative half-orbit $O^{-}(p)$ at the point $p$ is called a set

$$
\begin{aligned}
W^{u}\left(O^{-}(p)\right)= & \left\{x \in M: \exists O^{-}(x), \varrho\left(p_{-i} ; x_{-i}\right) \rightarrow 0 \text { as } i \rightarrow \infty,\right. \\
& \text { where } \left.p_{-i} \in O^{-}(p), x_{-i} \in O^{-}(x)\right\},
\end{aligned}
$$

which can be represented in the form

$$
W^{u}\left(O^{-}(p)\right)=\bigcup_{i \geqslant 0} f^{i}\left(W_{\delta, l o c}^{u}\left(O^{-}\left(p_{-i}\right)\right)\right) \text {, where } p_{-i} \in O^{-}(p) .
$$

Stable and unstable manifolds are called invariant manifolds.

We will need some auxiliary statements of a topological nature.

Lemma 1. Let $X$ be a topological space and $\mathcal{B}$ its base of topology consisting of connected sets. Let $N \subset X$ be a closed subset. Then each connected component of the complement $X \backslash N$ is an open set.

Proof. Consider an arbitrary point $x \in X \backslash N$. Since $X \backslash N$ is an open set, there is a connected open set $U \in \mathcal{B}$ such that $x \in U$. Let $K$ be a connected component $X \backslash N$ containing the point $x$. Since $K$ is the largest connected subset of $X \backslash N$ containing the point $x$, and $U$ is connected, the inclusion of $U \subset K$ takes place. The proved statement now immediately follows from the arbitrariness of the choice of the point $x$.

Corollary 1. Let $M$ be a closed manifold, and $N \subset M$ be its closed subset. Then each connected component of $M \backslash N$ is an open set.

Proof. Let us take a topology base $\mathcal{B}$ for the manifold $M$ as the union of open sets $\varphi\left(B_{r}(p)\right)$, where $\varphi: \mathbb{R}^{n} \rightarrow M$, a coordinate map of atlas of manifolds $M$ and $B_{r}(p)$, is the open ball of radius $r$. Now the required statement follows from Lemma 1.

Lemma 2. Let $M$ be a simply connected topological space, and let the continuous map $f: M \rightarrow M$ be a cover (in particular, a local homeomorphism). Then $f$ is a homeomorphism.

Proof.

Suppose that there are two different points $x_{1}, x_{2} \in M, x_{1} \neq x_{2}$, such that $f\left(x_{1}\right)=f\left(x_{2}\right)=$ $=x_{0}$. Let $G_{0}:[0 ; 1] \rightarrow M$ be a mapping of the segment $[0 ; 1]$ such that $G_{0}(0)=x_{1}, G_{0}(1)=x_{2}$. Then the mapping $F_{0}=f \circ G_{0}$ has the property $F_{0}(0)=F_{0}(1)$. As $M$ is simply connected, there is a homotopy $F_{t}:[0 ; 1] \rightarrow M, 0 \leqslant t \leqslant 1, F_{1}([0 ; 1])=x_{0}$. Since $f$ is a covering, by virtue of the covering homotopy theorem, there exists a homotopy $G_{t}:[0 ; 1] \rightarrow M, 0 \leqslant t \leqslant 1$, such that $F_{t}=f \circ G_{t}$ for all $0 \leqslant t \leqslant 1$. Therefore, $F_{1}([0 ; 1])=f \circ G_{1}([0 ; 1])=f\left(x_{1}\right)=f\left(x_{2}\right)$. Hence, the path $G_{1}([0 ; 1])$ connects in $f^{-1}\left(x_{0}\right)$ various points $G_{0}(0)=x_{1}, G_{0}(1)=x_{2}$, which contradicts the fact that the set $f^{-1}\left(x_{0}\right)$ consists of isolated points. The resulting contradiction proves the required statement, since $f$ is a local homeomorphism. 
Let us recall the definition of lamination. A detailed explanation of the theory of laminations can be found in [1]. $C^{r, l}$, a smooth $d$-dimensional lamination on an $n$-dimensional manifold $M^{n}(1 \leqslant d \leqslant n-1,0 \leqslant l \leqslant r \leqslant \infty)$, is a closed set $\mathcal{L} \subset M^{n}$, which is the union of $\bigcup_{\alpha} L_{\alpha}$ pairwise disjoint images of $L_{\alpha}$ of some $d$-dimensional manifolds with respect to some $C^{r}$-smooth injective immersion, and for any point $x \in \mathcal{L}$ there is its neighborhood $U(x)$ and $C^{l}$-diffeomorphism $\psi: U(x) \rightarrow \mathbb{R}^{n}$ such that any connected component of the intersection $U(x) \cap L_{\alpha}$ is mapped by a diffeomorphism $\psi$ to an open subset of a $d$-dimensional subspace $\left\{\left(x_{1}, \ldots, x_{n}\right) \in \mathbb{R}^{n} \mid x_{d+1}=c_{d+1}, \ldots, x_{n}=c_{n}\right\}$, while the restriction of $\psi$ on any connected component of the intersection of $U(x) \cap L_{\alpha}$ is a $C^{r}$-diffeomorphism on the image.

\section{Proof of the main results}

Proof of Theorem 1. The existence of an A-endomorphism $f: \mathbb{T}^{2} \rightarrow \mathbb{T}^{2}$ of torus $\mathbb{T}^{2}$ with a Cantor type one-dimensional contracting repeller satisfying Theorem 1 was proved in the work [8] of V.Z. Grines, E. V.Zhuzhoma and E. D. Kurenkov, see also [10]. The proof consists in constructing the so-called Smale surgery operation [21] for the algebraic Anosov endomorphism $\mathbb{T}^{2} \rightarrow \mathbb{T}^{2}$

For the sphere $\mathbb{S}^{2}$, the proof is based on the method of constructing the Plykin attractor on $\mathbb{S}^{2}$ using a two-leaf branched cover $\mathbb{T}^{2} \rightarrow \mathbb{S}^{2}$, see [16]. Consider the transformation group $\Gamma$ of the plane $\mathbb{R}^{2}$ generated by the group $\mathbb{Z}^{2}$ of integer shifts $x \mapsto x+m, y \mapsto y+k, m, k \in \mathbb{Z}$, and the symmetry $(x ; y) \mapsto(-x ;-y)$. Then the quotient space $\mathbb{R}^{2} / \Gamma$ is the two-dimensional sphere $\mathbb{S}^{2}$, and the natural projection $p: \mathbb{R}^{2} \rightarrow \mathbb{R}^{2} / \Gamma=\mathbb{S}^{2}$ is a branched cover with four branching points $\sigma_{1}, \sigma_{2}, \sigma_{3}, \sigma_{4} \in \mathbb{S}^{2}$ of index two each. The set

$$
\Sigma=\bigcup_{(m ; k) \in \mathbb{Z}^{2}}\{(m ; k)\} \cup\left\{\left(m+\frac{1}{2} ; k+\frac{1}{2}\right)\right\} \subset \mathbb{R}^{2}
$$

is the preimage of the set $\Sigma_{0}=\left\{\sigma_{1}, \sigma_{2}, \sigma_{3}, \sigma_{4}\right\}$, that is, $p(\Sigma)=\Sigma_{0}=\bigcup_{i=1}^{4} \sigma_{i}$.

It is known that, if the transformation $\tilde{f}: \mathbb{R}^{2} \rightarrow \mathbb{R}^{2}$ satisfies the relations

$$
\tilde{f}(\Sigma)=\Sigma, \quad \tilde{f} \circ \Gamma=\Gamma \circ \widetilde{f}
$$

then $\tilde{f}$ induces a transformation of $f: \mathbb{S}^{2} \rightarrow \mathbb{S}^{2}$ such that $f \circ p=p \circ f$.

Consider the linear transformation $A: \mathbb{R}^{2} \rightarrow \mathbb{R}^{2}$, defined by the matrix $A=\left(\begin{array}{ll}5 & 6 \\ 2 & 3\end{array}\right)$. We denote the matrix and the transformation with one letter, which should not lead to confusion. It is not difficult to check that $A$ satisfies the relations (3.1), and therefore $A$ induces the transformation $\mathbb{S}^{2} \rightarrow \mathbb{S}^{2}$, which we denote by $f_{0}$ and which has four fixed points $\sigma_{1}, \sigma_{2}, \sigma_{3}, \sigma_{4} \in \mathbb{S}^{2}$. The following construction applies to each of these points.

For simplcity, we will consider the point $\sigma_{1}=\sigma=p(0,0)$ (and omit the index). The point $\sigma$ has a single preimage $\widetilde{\sigma}(0,0) \in \mathbb{R}^{2}, p(\widetilde{\sigma})=\sigma$, such that $A(\widetilde{\sigma})=\widetilde{\sigma}$. Since $\sigma$ is a branching point of index two, there are neighborhoods $\widetilde{U}, U$ of points $\widetilde{\sigma}$ and $\sigma$, respectively, such that $p(\widetilde{U})=U$ and the restriction $\left.p\right|_{\widetilde{U}}: \widetilde{U} \rightarrow U$ is a natural projection of factor space $\widetilde{U} / s_{0} \rightarrow U$, where $s_{0}$ is the symmetry $(x ; y) \mapsto(-x ;-y)$. It is not difficult to verify that $\widetilde{\sigma}$ is a hyperbolic saddle point of the linear transformation $A$ with the eigenvectors $\vec{e}_{u}, \vec{e}_{s}$ corresponding to the eigenvalues $\lambda_{u}>1$ and $0<\lambda_{s}<1$, respectively. We will consider the pair $\vec{e}_{u}, \vec{e}_{s}$ as the basis (unit) vectors of 
the coordinate system $\left(v_{1} ; v_{2}\right)$ so that $(1 ; 0)$ and $(0 ; 1)$ are the coordinates $\vec{e}_{u}, \vec{e}_{s}$, respectively, and $\widetilde{\sigma}(0 ; 0)=O$.

For the set $N \subset \mathbb{R}^{2}$, we denote by $\mathbb{Z}^{2}(N)$ the family of sets obtained by the action of the group $\mathbb{Z}^{2}$ on $N$ (sets from $\mathbb{Z}^{2}(N)$ are called congruent to the set $N$ ).

Let us take $r_{0}>0$ so small that the $\lambda_{u} r_{0}$-neighborhood $\widetilde{U}_{0}$ of the point $O$ belongs to $\widetilde{U}$ and $\lambda_{u} r_{0}$-neighborhoods of all points from the set $p^{-1}\left(\sigma_{1} \cup \sigma_{2} \cup \sigma_{3} \cup \sigma_{4}\right)$ have no intersection. Let $\delta:[0 ; \infty) \rightarrow[0 ; 1]$ be a $C^{\infty}$-function such that $\delta(r) \equiv 1$ when $0 \leqslant r \leqslant \frac{r_{0}}{2}$, and $\delta(r) \equiv 0$ for $r \geqslant r_{0}$. Consider a system of differential equations in the neighborhood $\widetilde{U}_{0}$ and in all congruent to $\widetilde{U}_{0}$ neighborhoods, assuming that the coordinate system in $\widetilde{U}_{0}$ is transferred to the congruent neighborhood by the corresponding integer shift.

$$
\left\{\begin{array}{l}
\dot{v}_{1}=-v_{1} \cdot \ln \lambda_{u} \cdot \delta\left(\sqrt{v_{1}^{2}+v_{2}^{2}}\right) \\
\dot{v}_{2}=0 .
\end{array}\right.
$$

Denote by $\varphi_{r}^{t}$ the $t$-time shift along the trajectories of the system (3.2). Let us fix some number $t_{0}>1$, and put

$$
\widetilde{f}_{r}=A \circ \varphi_{r}^{t_{0}} .
$$

Since the system (3.2) is invariant under the symmetry of $\left(v_{1} ; v_{2}\right) \mapsto\left(-v_{1} ;-v_{2}\right)$, the diffeomorphism $\tilde{f}_{r}$ of the form (3.2) satisfies equation (3.1), and hence induces an endomorphism of $f_{r}: \mathbb{S}^{2} \rightarrow \mathbb{S}^{2}$. Using a method completely analogous that of [8], one can show that $f_{r}$ is an $A$-endomorphism and the nonwandering set of $f^{r}$ has a strictly invariant one-dimensional and uniquely hyperbolic contracting repeller $\Lambda_{r}=\Omega$, locally homeomorphic to the product of the segment and the Cantor set. In this case, any connected component of the set $\mathbb{S}^{2} \backslash \Lambda_{r}$ has a boundary admissible from $\mathbb{S}^{2} \backslash \Lambda_{r}$, which consists of exactly one stable manifolds of periodic points from $\Lambda_{r}$.

Proof of Theorem 2. Let us show that the basic set $\Lambda$ cannot be an expanding attractor. Let us assume the contrary. Then there is an $A$-endomorphism $f: M^{2} \rightarrow M^{2}$ with a Cantor type expanding attractor $\Lambda$ such that any connected component of the set $M^{2} \backslash \Lambda_{a}$ has a boundary admissible from $M^{2} \backslash \Lambda$ consisting of a finite number of unstable manifolds of points from $\Lambda_{a}$. Consider an arbitrary point $x \in \Lambda$. Let us show that the restriction $\left.f\right|_{W^{u}(x)}: W^{u}(x) \rightarrow f\left(W^{u}(x)\right)=$ $=W^{u}(f(x))$ of an endomorphism $f$ on any unstable manifold $W^{u}(x), x \in \Lambda$, is a homeomorphism in the intrinsic topology of the leaf $W^{u}(x)$.

First, we note that due to the hyperbolicity of $\Lambda$ the restriction $f$ on the set $\Lambda$ is a local diffeomorphism. Since $\Lambda$ is an attractor, it follows by virtue of Theorem 1 from [9] that $W^{u}(x)$ belongs to $\Lambda$. Therefore, $W^{u}(x)$ does not contain branch points of the endomorphism $f$ and, therefore, the restriction $\left.f\right|_{W^{u}(x)}$ is a cover without branch points. Now the required statement follows from the fact that $W^{u}(x)$ is simply connected and from Lemma 2 .

We now show that in the complement $M^{2} \backslash \Lambda$ there is some periodic point $O$ of the endomorphism $f$. Since $\Lambda$ is an attractor, there is a closed neighborhood $U \supset \Lambda$ such that $f(U) \subset \operatorname{int}(U)$, and $\bigcap_{l=0}^{\infty} f^{l}(U)=\Lambda$. Consider an arbitrary point $y_{0} \in M^{2} \backslash U$ and its arbitrary negative semiorbit $O^{-}\left(y_{0}\right)$. It follows directly from the inclusion of $f(U) \subset U$ that the intersection $O^{-}\left(y_{0}\right) \cap U$ is empty. Since the $\alpha$-limit set of any point $x \in M^{2}$ is contained in the nonwandering set $N W(f)$, the closure $\operatorname{clos}\left(O^{-}\left(y_{0}\right)\right)$ contains a point $w \in N W(f) \backslash \Lambda$. Then, by virtue of the spectral decomposition theorem, there is a basic set $\Lambda_{1} \subset M^{2} \backslash U$ different from $\Lambda$ containing the point $w$. 
In the basic set $\Lambda_{1}$, we choose an arbitrary periodic point $O$ of some smallest period $k$. If $k>1$, then further arguments can be carried out to map $f^{k}$. Thus, below it is possible to assume that point $O$ is a fixed point of the $f$ (without loss of generality).

Let $K$ be the connected component of the complement $M^{2} \backslash \Lambda$ containing the fixed point $O$. Denote by $\delta(K)$ the admissible boundary of the $K$ from interior $K$ and take a leaf $l \subset \Lambda$ of the lamination $\Lambda$ such that $l$ belongs to $\delta(K)$. By the condition, $l$ is an unstable manifold of some point from $\Lambda$. We show that $f^{r}(l)=l$ for some $r \in \mathbb{N}$.

Indeed, recall that, as $l$ belongs the admissible boundary from interior $K$, for any point $z \in l$ there exists an arc $d$ with endpoints $O$ and $z$ such that $d \backslash\{z\} \subset K$. Since $\Lambda$ is strictly invariant with respect to $f$, the point $f(z)$ belongs to $\Lambda$, and $f(d) \backslash\{f(z)\}$ belongs to $M^{2} \backslash \Lambda$. Since $f(O)=$ $=O$, it follows that $f(d) \backslash\{f(z)\}$ belongs to $K$. Therefore, $f(z)$ belongs to the leaf $f(l)$ of lamination $\Lambda$ such that $l \subset \delta(K)$. This means that the set $\delta(K)$ is invariant. Since $\delta(K)$ consists of a finite number of leaves, $f^{r}(l)=l$ for some $r \in \mathbb{N}$. Taking the iterations $f^{n r}$, if necessary, we will assume below that all leaves of the lamination $\Lambda$ belonging to $\delta(K)$ are invariant with respect to $f$.

Consider an arbitrary point $p \in l$, where $l \in \delta(K)$. Since the restriction of $f$ to $\Lambda$ is a $k$-cover, $k \geqslant 2$, the complete preimage of $f^{-1}(p)$ contains some point $p_{1} \neq p$. Since $f(\Lambda)=\Lambda=$ $=f^{-1}(\Lambda), p_{1} \in \Lambda$. Due to the injectivity of the restriction $\left.f\right|_{W^{u}(p)}$, we have that $p_{1} \notin l$. It is clear that $p_{1}$ does not belong to the boundary $\delta(K)$ admissible from $K$. Indeed, suppose $p_{1} \in l_{*}$ where $l_{*}$ is the leaf of the lamination $\Lambda$ admissible from $K$. The leaf $l_{*}$ is invariant respect to $f$, different from $l$ and $f\left(l^{*}\right)=l$, which is impossible.

Consider the path $\gamma_{p}$ connecting the points $p$ and $O$ such that int $\gamma_{p} \in K$. Since $p_{1} \neq p$, there is a path $\gamma_{p}^{\prime}$ connecting the points $p_{1}$ and $O_{1}$, such that $\gamma_{p}=f\left(\gamma_{p}^{\prime}\right), f\left(O_{1}\right)=O$. Thus, $p_{1}$ belongs to the admissible boundary of some connected component $K_{1}$ of the set $M^{2} \backslash \Lambda$. Moreover, $p_{1}$ belongs to a leaf, say $l_{1}$, of the lamination $\Lambda$ such that $f\left(l_{1}\right)=l$. Since $l_{1} \notin \delta(K), K \neq K_{1}$. Similarly, it is shown that the complete preimage of $f^{-1}\left(p_{1}\right)$ contains a point $p_{2} \notin\left\{p, p_{1}\right\}$ belonging to the component $K_{2}$ of the set $M^{2} \backslash \Lambda \mathrm{t}$ such that $K_{2} \notin\left\{K, K_{1}\right\}$, and there is a point $O_{2} \in K_{2}$ such that $f\left(O_{2}\right)=O_{1}$. Continuing this process, we get a negative semiorbit $O^{-}(p)=\left\{p=p_{0}, p_{1}, p_{2}, \ldots\right\}, f\left(p_{j+1}\right)=p_{j}$, and the sequence $\left\{K=K_{0}, K_{1}, K_{2}, \ldots\right\}$, $f\left(K_{j+1}\right)=K_{j}$, with the following properties:

1) the point $p_{i}$ belongs to the boundary of the domain $K_{i}$ admissible from $K_{i}$ for all $i \in \mathbb{N} \cup\{0\}$;

2) $K_{i}$ are pairwise distinct connected components of the set $M^{2} \backslash \Lambda$;

3) there is a negative semi-orbit $O^{-}(O)=\left\{O, O_{1}, O_{2}, \ldots\right\}, f\left(O_{j+1}\right)=O_{j}$ such that $O_{i} \in K_{i}$ for all $i \in \mathbb{N} \cup\{0\}$.

Since $O \in M^{2} \backslash U$ and $f(U) \subset U$, there is an inclusion of $O^{-}(O) \subset M^{2} \backslash \operatorname{int}(U)$. Hence, taking into account the compactness of the set $M^{2} \backslash \operatorname{int}(U)$, it follows that the sequence $O^{-}(O)$ has a limit point $O^{*} \in M^{2} \backslash \operatorname{int}(U)$. Let $K^{*}$ be the connected component of the set $M^{2} \backslash \Lambda$ containing the point $O^{*}$. According to Corollary 1, the connected component $K^{*}$ is an open set. Then there are two different numbers $n_{1}, n_{2} \in \mathbb{N}$ such that $O_{n_{1}} \in K^{*}$ and $O_{n_{2}} \in K^{*}$, which contradicts the fact that $K_{n_{1}}$ and $K_{n_{2}}$ are different connected components of the set $M^{2} \backslash \Lambda$.

Proof of Theorem 3. Due to the conditions, the stable manifolds of the repeller $\Lambda_{r}$ form the lamination [15], which we denote by $\mathcal{L}\left(\Lambda_{r}\right)$. The set $M^{2} \backslash \mathcal{L}\left(\Lambda_{r}\right)$ is the union of a countable number of pairwise disjoint open disks that are basins of isolated sink periodic orbits. In addition, the lamination $\mathcal{L}\left(\Lambda_{r}\right)$ can be extended to some foliation $F\left(\Lambda_{r}\right)$ on the surface of $M^{2}$.

RUSSIAN JOURNAL OF NONLINEAR DYNAMICS, 2021, 17(3), 335-345 
It is known that the sum of the indices of singularites of a foliation is equal to the Euler characteristic of the ambient surface (see, for example, [1, 14]). Suppose that $M^{2} \neq \mathbb{T}^{2}, \mathbb{S}^{2}$. Since in this case the Euler characteristic of $M^{2}$ is negative, there is a component $C$ of the set $M^{2} \backslash \mathcal{L}\left(\Lambda_{r}\right)$ containing the singularity of index $\leqslant-\frac{1}{2}$. Denote by $\nu$ the number of leaves from $\delta(C)$. Since the index of the singularity from $C$ satisfies the inequality $1-\frac{\nu}{2} \leqslant-\frac{1}{2}$, we see that $\nu \geqslant 3$. It follows from the strict invariance of $\Lambda_{r}$ that $f^{-1}(\delta(C)) \subset \Lambda_{r}$. Moreover, since the restriction $\left.f\right|_{\Lambda_{r}}$ is a local diffeomorphism, the preimage $f^{-1}(\delta(C))$ belongs to the admissible boundary of some component of $M^{2} \backslash \mathcal{L}\left(\Lambda_{r}\right)$. Since each component of the set $M^{2} \backslash \mathcal{L}\left(\Lambda_{r}\right)$ is homeomorphic to an open disk, it is simply connected. Thus, $f^{-1}(\delta(C))$ is the union of the admissible boundaries of either one or several connected components of the set $M^{2} \backslash \mathcal{L}\left(\Lambda_{r}\right)$. In any case, since the lamination $\mathcal{L}\left(\Lambda_{r}\right)$ is regular, the preimage of $f^{-1}(C)$ contains a component of the set $M^{2} \backslash \mathcal{L}\left(\Lambda_{r}\right)$, different from $C$, with singularity of a negative index. Since $f$ is not a diffeomorphism, the number of singularities of the negative index must be infinitely large. Since the surface $M^{2}$ is compact, the foliation $F\left(\Lambda_{r}\right)$ must have only a finite number of singularities of the nonzero index, by virtue of the Euler - Poincaré formula (see, e. g., [1, 14]). The contradiction completes the proof.

Let us illustrate our arguments above for the simplest saddle-type singularities, when the set of singularities is invariant with respect to $f$. Let us assume for simplicity that the singularity $s_{0} \in C$ of the negative index has $\nu$ separatrix. Then its index ind $s_{0}$, according to [14], formula (3.11), equals $1-\frac{\nu}{2}$, where $\nu \geqslant 3$. If $f$ has no branch points in $C$, then the foliation $F\left(\Lambda_{r}\right)$ has in each component of $f^{-1}(C)$ a singularity of the index $1-\frac{\nu}{2}<0$. If $s_{0}$ is a branch point of degree $k \geqslant 2$, then each singularity of $f^{-1}\left(s_{0}\right)$ has $k \cdot \nu$ separatrix and therefore its index equals $1-\frac{k \cdot \nu}{2}<0$. In both cases, the index of a singularity from the preimage $f^{-1}\left(s_{0}\right)$ is negative.

Remark. The Euler characteristic of the surfaces $\mathbb{T}^{2}$ and $\mathbb{S}^{2}$ equals zero and two, respectively. Therefore, except for singularities of zero index, there are possible singularities of the index $\frac{1}{2}$ for $\nu=1$. However, their preimages for $k=2$ have zero index. This shows that, for the surfaces $\mathbb{T}^{2}$ and $\mathbb{S}^{2}$, the proof of Theorem 3 does not hold.

\section{References}

[1] Anosov, D. V. and Zhuzhoma, E. V., Nonlocal Asymptotic Behavior of Curves and Leaves of Laminations on Universal Coverings, Proc. Steklov Inst. Math., 2005, no. 2(249), pp. 1-219; see also: Tr. Mat. Inst. Steklova, 2005, vol. 249, pp. 3-239.

[2] Grines, V.Z., The Topological Equivalence of One-Dimensional Basic Sets of Diffeomorphisms on Two-Dimensional Manifolds, Uspekhi Mat. Nauk, 1974, vol. 29, no. 6(180), pp. 163-164 (Russian).

[3] Grines, V.Z., The Topological Conjugacy of Diffeomorphisms of a Two-Dimensional Manifold on One-Dimensional Orientable Basic Sets: 1, Tr. Mosk. Mat. Obs., 1975, vol. 32, pp. 35-60 (Russian).

[4] Grines, V.Z., The Topological Conjugacy of Diffeomorphisms of a Two-Dimensional Manifold on One-Dimensional Orientable Basic Sets: 2, Tr. Mosk. Mat. Obs., 1977, vol. 34, pp. 243-252 (Russian).

[5] Grines, V.Z., On the Topological Classification of Structurally Stable Diffeomorphisms of Surfaces with One-Dimensional Attractors and Repellers, Sb. Math., 1997, vol. 188, no.4, pp. 537-569; see also: Mat. Sb., 1997, vol. 188, no. 4, pp. 57-94.

[6] Grines, V.Z. and Zhuzhoma, E.V., The Topological Classification of Orientable Attractors on an $n$-Dimensional Torus, Russian Math. Surveys, 1979, vol. 34, no. 4, pp. 163-164; see also: Uspekhi Mat. Nauk, 1979, vol. 34, no. 4, pp. 185-186. 
[7] Grines, V.Z. and Zhuzhoma, E. V., On Rough Diffeomorphisms with Expanding Attractors or Contracting Repellers of Codimension One, Dokl. Math., 2000, vol.62, no. 2, pp. 274-276; see also: Dokl. Ross. Akad. Nauk, 2000, vol. 374, no. 6, pp.735-737.

[8] Grines, V.Z., Zhuzhoma, E.V., and Kurenkov, E.D., On DA-Endomorphisms of the TwoDimensional Torus, Sb. Math., 2021, vol.212, no.5, pp.698-725; see also: Mat. Sb., 2021, vol. 212, no. 5, pp. 102-132.

[9] Grines, V.Z. and Kurenkov, E. D., On Hyperbolic Attractors and Repellers of Endomorphisms, Nelin. Dinam., 2017, vol. 13, no. 4, pp. 557-571 (Russian).

[10] Kurenkov, E. D., On Existence of an Endomorphism of 2-Torus with Strictly Invariant Repeller, Zh. SVMO, 2017, vol. 19, no. 1, pp. 60-66 (Russian).

[11] Plykin, R. V., Sources and Sinks of A-Diffeomorphisms of Surfaces, Math. USSR-Sb., 1974, vol. 23, no. 2, pp. 233-253; see also: Mat. Sb. (N.S.), 1974, vol. 94(136), no. 2(6), pp. 243-264.

[12] Plykin, R. V., On the Geometry of Hyperbolic Attractors of Smooth Cascades, Russian Math. Surveys, 1984, vol. 39, no. 6, pp. 85-131; see also: Uspekhi Mat. Nauk, 1984, vol. 39, no.6(240), pp. 75113.

[13] Grines, V. and Zhuzhoma, E., On Structurally Stable Diffeomorphisms with Codimension One Expanding Attractors, Trans. Amer. Math. Soc., 2005, vol. 357, no. 2, pp. 617-667.

[14] Grines, V.Z. and Zhuzhoma, E. V., Surface Laminations and Chaotic Dynamical Systems, Izhevsk: R\&C Dynamics, Institute of Computer Science, 2021.

[15] Ikegami, G., Hyperbolic Sets and Axiom A for Endomorphisms, Proc. of Inst. of Natural Sciences, Nihon Univ., 1991, vol. 26, pp. 69-86.

[16] Katok, A., Bernoulli Diffeomorphisms on Surfaces, Ann. of Math. (2), 1979, vol. 110, no. 3, pp. 529547.

[17] Newhouse, S., On Codimension One Anosov Diffeomorphisms, Amer. J. Math., 1970, vol. 92, no. 3, pp. 761-770.

[18] Przytycki, F., Anosov Endomorphisms, Studia Math., 1976, vol. 58, no. 3, pp. 249-285.

[19] Przytycki, F., On $\Omega$-Stability and Structural Stability of Endomorphisms Satisfying Axiom A, Studia Math., 1977, vol. 60, no. 1, pp. 61-77.

[20] Shub, M., Endomorphisms of Compact Differentiable Manifolds, Amer. J. Math., 1969, vol. 91, no. 1, pp. 175-199.

[21] Smale, S., Differentiable Dynamical Systems, Bull. Amer. Math. Soc., 1967, vol.73, no. 6, pp. 747817. 